\title{
Effector-independent voluntary timing: behavioural and neuroimaging evidence
}

\author{
Sara L. Bengtsson, ${ }^{*}$ H. Henrik Ehrsson, ${ }^{*}$ Hans Forssberg and Fredrik Ullén \\ Neuropaediatric Research Unit, Department of Woman and Child Health, Karolinska Institutet, SE-171 76 Stockholm, Sweden
}

Keywords: auditory-motor interaction, fMRI, rhythm, superior temporal gyrus, supplementary motor area

\begin{abstract}
We investigated effector-independent aspects of voluntary motor timing, using behavioural measurements and functional magnetic resonance imaging. Two types of temporal pattern were investigated; one isochronous, the other a metric, rhythmic sequence of six temporal intervals. Each pattern was performed using tapping movements with the left or right index fingers, or rhythmic speech on one syllable. Deviations from the ideal temporal pattern in the rhythmic sequence tasks were consistent between the three different effectors, within subjects. This suggests that the same representation of the rhythm was used to time the movements with all effectors. To reveal brain regions involved in such effector-independent timing, we localized the overlap in brain activity when the rhythmic sequence was performed with the different effectors. Activity was found in the mesial and lateral premotor cortices, posterior and anterior regions of the superior temporal gyrus and the inferior frontal cortex. Subcortical activations were in the left globus pallidus, the vermis and bilaterally in the cerebellar hemispheres (lobule $\mathrm{VI}$ ) and the thalamus. The overlap in activity between the isochronous tasks included the same set of brain regions, except for the basal ganglia and the thalamus. Rhythmic sequences had significantly higher activity in mesial premotor cortex, the left superior temporal gyrus and the cerebellum, than had isochronous movements. These findings reveal a set of brain regions likely to be involved in effector-independent representations of temporal patterns in voluntary motor timing. A subset of these regions plays important roles for the organization of rhythmic sequences of several intervals.
\end{abstract}

\section{Introduction}

Many skills, such as speaking, typing, and playing a musical instrument, can be regarded as rhythmic sequences of individual movements. To perform such behaviours, the brain must control both the serial order of the individual movements and their timing (Lashley, 1967; Martin, 1972; Keele \& Ivry, 1987). Here, we focus on mechanisms for voluntary timing. For most everyday skills, this involves relatively short $(\sim 200$ $2000 \mathrm{~ms}$ ) temporal intervals (Wing, 2002). We will use the term temporal structure for the pattern of temporal intervals between the movements of a sequence. The simplest temporal structure is the isochronous rhythm, where all temporal intervals are equal. More complex temporal structures form rhythmic sequences of several temporal intervals, not all of the same duration.

The main aim of this study was to characterize brain regions involved in effector-independent aspects of motor timing. We examined both isochronous temporal patterns and rhythmic sequences. The first indications of effector-independent mechanisms in temporal control came from studies showing positive within-subject correlations for the maximum rate (Keele \& Hawkins, 1982) and temporal variability (Franz et al., 1992; Robertson et al., 1999) of isochronous movements performed with different effectors. Additional evidence that the brain can use movement-independent representations of temporal patterns to time performance has come from studies on

Correspondence: Dr Fredrik Ullén, as above.

E-mail: Fredrik.Ullen@ki.se

*Present address: Wellcome Department of Imaging Neuroscience, Institute of Neurology, London, UK.

Received 20 April 2005, revised 1 November 2005, accepted 2 November 2005 spatiotemporal sequences performed with the same effector (Ullén \& Bengtsson, 2003; Bengtsson et al., 2004a). In these studies, it was found that the temporal pattern of a movement sequence could be learned and executed independently of its spatial aspects. Furthermore, when the spatial structure of the sequence was varied, the details of the performed temporal pattern remained highly consistent within subjects.

As model behaviours we used finger tapping, which has been frequently employed in studies of voluntary timing (Wing, 2002), and rhythmic speech. Isochronous tapping has been extensively studied in synchronization/continuation experiments (Wing \& Kristofferson, 1973). In that paradigm, the subject first taps in synchrony with a series of rhythmic stimuli; after the last stimulus, the subject continues with self-paced tapping using the same temporal structure. Both the synchronization and continuation phases activate an extensive network of brain regions. Typically, active areas include contralateral primary sensorimotor cortex (M1/S1), the supplementary (SMA) and presupplementary (preSMA) motor areas, the dorsal (PMD) and ventral (PMV) premotor cortices, the inferior frontal gyrus (IFG), the superior temporal gyrus (STG), the cerebellum, the thalamus and the basal ganglia (Rao et al., 1997; Jäncke et al., 2000; Lutz et al., 2000; Rubia et al., 2000; Jantzen et al., 2004, 2005).

Rhythmic sequence performance with high accuracy usually requires training. During the initial stages of rhythmic sequence learning, brain activity increases in regions that are also active during isochronous tapping, including the SMA/preSMA, the PMD, the PMV, and the cerebellum (Ramnani \& Passingham, 2001; Sakai et al., 2002). Well-learned rhythmic sequence performance has been found to activate the SMA/preSMA, the PMV, the inferior frontal cortex, and 
the superior temporal cortex. These regions were involved in the control of the rhythmic sequence, independently of its spatial structure (Bengtsson et al., 2004a). Recently, it was demonstrated that the mesial and lateral premotor cortices, as well as the basal ganglia, increase their activity parametrically with sequence length, during the initiation phase of performance of well-learned rhythmic sequences (Lewis et al., 2004).

The picture emerging from these neuroimaging studies is largely consistent with neurological data and data from experiments with transcranial magnetic stimulation (TMS). Patients with lesions to the mesial premotor cortex or the PMD show impaired production of rhythmic sequences (Halsband et al., 1993). Impaired movement timing after cerebellar damage has been demonstrated in several studies (Ivry et al., 1988; Ivry \& Keele, 1989; Spencer et al., 2003; Harrington et al., 2004), and includes performance with different effectors (Ivry et al., 1988). Increased variability of isochronous finger tapping has been reported after repetitive TMS of the cerebellum (Théoret et al., 2001).

In this study, we first used the approach of Ullén \& Bengtsson (2003) to test if participants show high intrasubject consistency of the temporal pattern, when performing the same well-learned rhythmic sequence with the left index finger, the right index finger and using rhythmic speech. This hypothesis was confirmed by the behavioural data (see Results), suggesting that a common, effector-independent representation of the rhythmic sequence was used to time the movements in each case.

Secondly, we used fMRI to identify candidate brain regions for effector-independent temporal control. The participants performed isochronous movements, as well as the same rhythmic sequence as in the behavioural experiment, with the three different effectors; a difference was that silent speech (imagery) was used instead of overt speech to avoid movement artefacts. Isochronous and rhythm sequence performance were contrasted with rest for each effector, and conjunction analyses of these contrasts were employed to reveal overlap in activity, which is likely to include areas involved in effector-independent control of each temporal pattern. Finally, we investigated if the preSMA/SMA, the lateral premotor cortex, the superior temporal cortex, the basal ganglia and the cerebellum, which we earlier found to be specifically involved in the control of the temporal pattern of a spatiotemporal sequence (Bengtsson et al., 2004a), are involved in effector-independent aspects of rhythmic sequence control. For this purpose, we compared rhythmic sequence performance with isochronous performance, across the three effectors, using a conjunction analysis.

\section{Materials and methods}

\section{Subjects}

Seven healthy, right-handed (Oldfield, 1971) subjects (4 males and 3 females) with no history of neurological disease participated in the study. No subjects were professional musicians or music students. Subjects were between 22 and 28 years old, with a mean age of 24.3 years \pm 2.1 (standard deviation, SD). All subjects had given informed consent to participate in the study, and the experimental procedures were ethically approved by the Karolinska Institutet Ethical Committee (KI Forskningsetikkommitté Nord; Dnr 00-195).

\section{Behavioural tasks}

Seven conditions were used; six experimental tasks and one baseline condition, REST, where the subjects relaxed without any active movement. In three of the experimental conditions, SEQRIGHT, SEQLEFT, and SEQSPEECH, the subjects performed a rhythmic sequence of six temporal intervals, with the right index finger, the left index finger, and using rhythmic speech, respectively (Fig. 1, upper half). The rhythmic sequence had the temporal pattern 375-375-7501125-375-1500 (all in $\mathrm{ms}$ ), and a total duration of $4500 \mathrm{~ms}$. This sequence can be divided into three metric units, each $1500 \mathrm{~ms}$ long. The onset times of these units are marked with vertical arrows in Fig. 1. In the other three experimental conditions, ISORIGHT, ISOLEFT and ISOSPEECH, the subjects performed an isochronous rhythm (interval between taps: $750 \mathrm{~ms}$ ) with the same effectors (Fig. 1, lower half). The mean interval between movements in the sequential and isochronous conditions was the same $(750 \mathrm{~ms})$, and all conditions were thus matched in terms of the total number of movements performed (Fig. 1).

In those tasks where index finger movements were used, the subjects performed brisk, rhythmic tapping movements on a nonmagnetic optic force transducer. The vertical tap forces were acquired, displayed on-line, and recorded on a PC using the SC/ZOOM data acquisition system (Department of Physiology, University of Umeå, Sweden) with a sampling frequency of $0.8 \mathrm{kHz}$. In the speech conditions (SEQSPEECH and ISOSPEECH) subjects performed rhythmic speech on the syllable 'pa'. During the training session (see below) subjects used overt speech, which was recorded (Fig. 1) and analysed. Due to a technical failure, speech training data was not properly recorded from one subject, and the correlational analyses of consistency in timing between effectors (see Results) were thus performed on the remaining six subjects. To avoid movement artefacts, subjects instead used silent speech (motor imagery) without any active movements during the fMRI scans.

\section{Training of the tasks}

Each subject practised all tasks in one session (1-h long), 1-3 days before the experiment. The tasks were practised in 40-s long epochs, as during the scanning, in two different ways. In one case, subjects heard the temporal pattern of the task, presented repetitively through headphones as a recorded sequence of drumbeats, and performed the task in synchrony with these auditory stimuli. In the other case, subjects performed the task from memory, as they would during the scanning. During training, epochs where the subject synchronized with an auditory presented temporal pattern, and epochs where the subject performed from memory were alternated. All tasks were rehearsed an approximately equal amount of time. The subjects were after this training session able to perform all tasks robustly while simultaneously maintaining a conversation with the experimenter. One additional shorter $(30 \mathrm{~min})$ rehearsal was performed immediately before the MR recording, where it was verified that the subject was able to perform all tasks from memory while talking to the experimenter.

\section{Experimental procedure}

During the MR scans, subjects rested comfortably in a supine position in the MR scanner with the arms extended parallel to the trunk. All tasks were performed in epochs lasting $40 \mathrm{~s}$. During the first $8 \mathrm{~s}$ of each epoch, the subjects were instructed which task to perform. This instruction consisted of a verbal cue (the name of the condition), followed by five isochronous beats of an auditory metronome with an interstimulus interval of $750 \mathrm{~ms}$, which gave the subjects the correct tempo. The subjects started to tap in phase with the metronome, and 


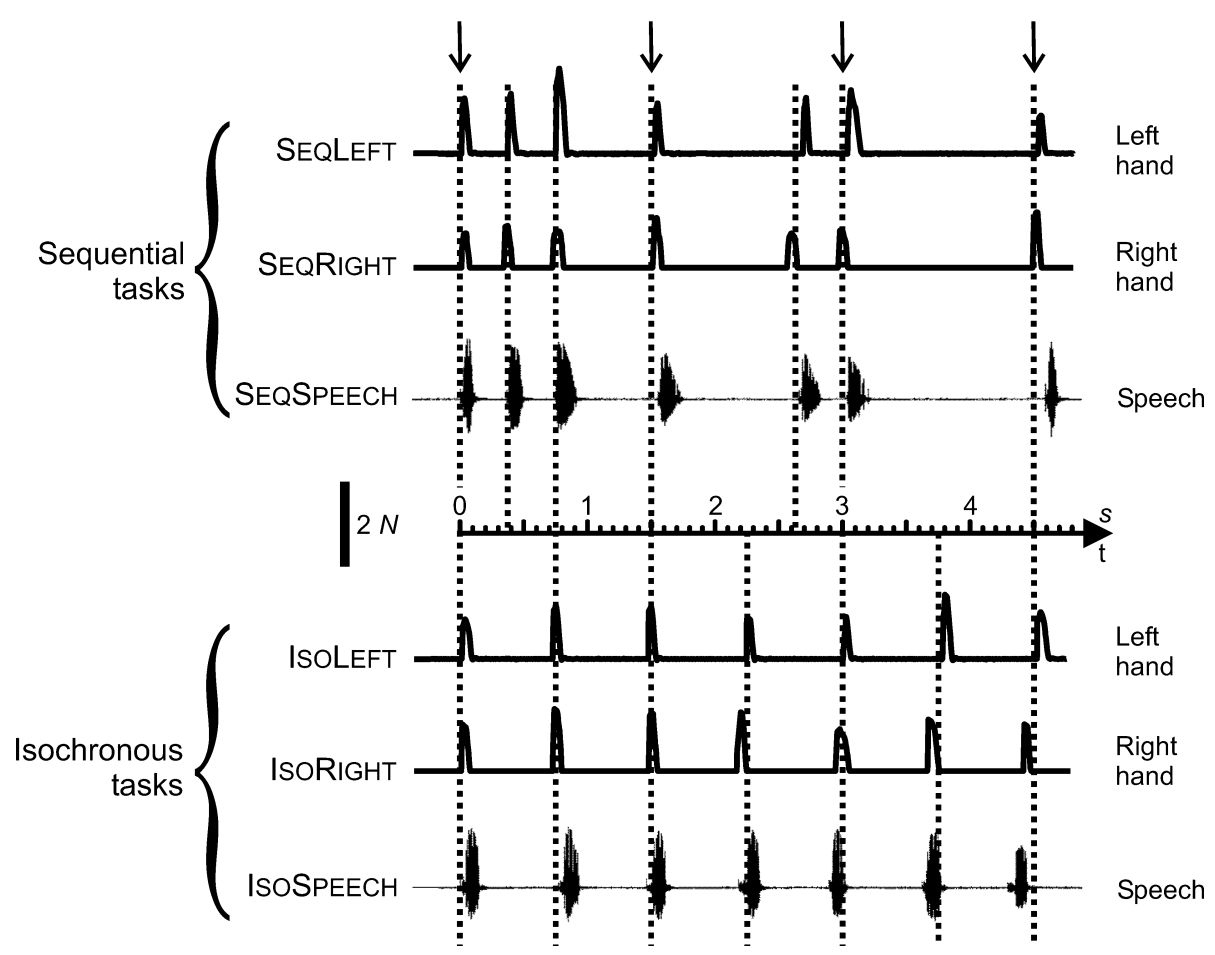

FIG. 1. Behavioural tasks. Typical examples of tapping force recordings and voice recordings for the different conditions, from a single subject. Tapping force recordings are from the fMRI scanning period, whereas voice recordings are from the training where overt speech was used. Vertical dashed lines illustrate the ideal temporal pattern for the sequential (upper half) and isochronous (lower half) tasks. Vertical arrows in the upper part show the onsets of metrical units (see text).

thereafter continued with self-paced, repetitive performance of the task for the remaining $32 \mathrm{~s}$ of the epoch. Data from these $32 \mathrm{~s}$ was used in the subsequent analysis. The rhythmic sequence conditions were repeated continuously without any breaks between cycles.

\section{Data acquisition: $f M R I$}

Functional magnetic resonance imaging (fMRI) was conducted on a $1.5 \mathrm{~T}$ scanner (Signa Horizon Echospeed, General Electric Medical Systems, Milwaukee, WI, USA). At the beginning of each experiment a high-resolution, three-dimensional gradient echo T1-weighted anatomic image volume of the whole brain (voxel size $1 \times 1 \times 1 \mathrm{~mm}$ ) was collected. Functional imaging data was then recorded as gradient-echo, echo-planar (EPI) T2*-weighted images with blood oxygenation level dependent (BOLD) contrast (Kwong et al., 1992; Ogawa et al., 1992; Logothetis, 2002). Image volumes of the whole brain were built up from contiguous axial slices $(n=30)$. The following parameter values were used for the fMRI scanning: echo time, $60 \mathrm{~ms}$; field of view, $22 \mathrm{~cm}$; matrix size, $64 \times 64$; pixel size, $3.4 \mathrm{~mm} \times 3.4 \mathrm{~mm}$; flip angle, $90^{\circ}$; slice thickness, $5.0 \mathrm{~mm}$; repetition time $\left(T_{R}\right), 4 \mathrm{~s}$; number of volumes per run, 122. The image volumes were collected continuously during separate runs, where all tasks were performed twice in the same order in each run. To reduce possible time and order effects three different task-orders were used for different runs. We started each run by recording four 'dummy' image volumes that were not stored, to allow for T1 equilibration effects. Six runs were recorded from each subject.

\section{Image processing and data analysis}

Functional MR data was analysed using the SPM-99 software package (Wellcome Department of Imaging Neuroscience, London, UK). The volumes were realigned to correct for head movements. Subsequently, the volumes were coregistered to each individual's T1-weighted image and normalized to the stereotactic coordinate system of Talaraich \& Tournoux (1988), using the template brain of the Montréal Neurological Institute. Proportional scaling was applied to eliminate the effects of global changes in the signal. The time series were smoothed spatially with an isotropic Gaussian filter of $8 \mathrm{~mm}$ full width at half-maximum, and temporally with a Gaussian kernel of width $4 \mathrm{~s}$. The fMRI data was modelled with a standard linear regression model, as implemented in SPM-99, where we defined seven conditions of interest corresponding to the periods in each epoch when the subjects performed the tasks (or rested) without hearing the metronome. Seven conditions of no interest were also modelled, corresponding to the first $8 \mathrm{~s}$ of each epoch when the subjects were listening to the verbal cue and the metronome. Condition-dependent activations were modelled with a boxcar waveform, convolved with a haemodynamic response function. The significance of the effects was assessed using $t$ statistics for every voxel from the brain to create statistical parametric maps (SPMs), which were subsequently transformed into $Z$ statistics. To increase the sensitivity of the analysis, we pooled the data from all subjects, performing a group analysis. Thus the statistical analyses used in the present study are fixed-effect analyses. This implies that our inferences pertain only to the subjects included in the present study. Peaks of activity are reported, when significant at $P<0.05$, using single voxel based statistics (Friston et al., 1995) with corrections for the total number of comparisons in the whole brain volume when contrasting the isochronous and sequential tasks with rest. For the contrast between sequential and isochronous conditions we had an a priori hypothesis from our previous imaging study (Bengtsson et al., 2004a) of greater activity in the preSMA/SMA, the lateral premotor cortex, the superior temporal cortex, the basal ganglia, and the cerebellum. Therefore, we used small volume corrections for multiple comparisons within spherical regions 
of interest with radii of $10 \mathrm{~mm}$ in these regions. The coordinates for the centre of the spheres were taken from the peaks of activity, which were detected in the main effect contrast for rhythmic sequence control (long temporal structure) in Bengtsson et al. (2004a). A region of interest analysis was only employed in the sequential vs. isochronous contrast; for the other contrasts a whole brain analysis was used.

The focus of the present study was to investigate brain regions involved in different aspects of effector-independent control of motor timing. For this purpose, we used 'minimum statistic compared to the conjunction null' analyses, as described in Nichols et al. (2005). This conjunction analysis can be interpreted as a logical AND-operation, giving only those activations that are significant in all individual contrasts of the conjunction.

First, the conjunction between the three contrasts SEQRIGHT - REST, SEQLEFT - REST, and SEQSPEECH - REST was calculated. The conjunction analysis will show only those regions that are activated in all three contrasts, which is likely to include regions controlling effector-independent aspects of the performance. Secondly, using the same logic, we calculated the conjunction between the contrasts ISORIGHT - REST, ISOLEFT - REST and ISOSPEECH - REST, in order to localize candidate brain regions involved in effector-independent aspects of isochronous performance. Finally, we investigated which brain regions show higher activity during rhythmic sequence performance than during isochronous performance, irrespective of effector. For this purpose we calculated the conjunction between SEQRIGHT - ISORIGHT, SEQLEFT ISOLEFT and SEQSPEECH - ISOSPEECH.

Anatomical localizations of the activated regions in terms of major sulci and gyri (Duvernoy, 2000) were determined from an average image of normalized and intensity standardized T1-weighted images from all seven subjects. Cerebellar activations were localized using the atlas of Schmahmann et al. (2000).

A

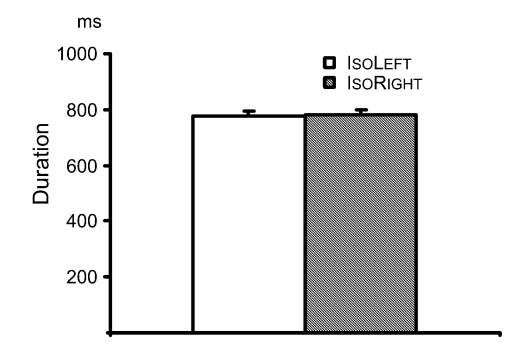

C

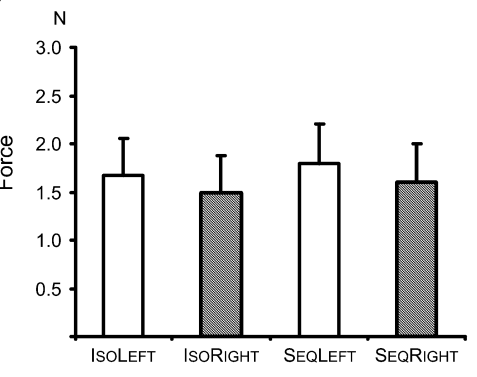

Results

\section{Behavioural data}

Task performance

The behavioural data consisted of tap force recordings of the tapping movements performed with the left and right hand during the scanning. In addition, rhythmic speech data was examined from the training sessions (Fig. 1). During scanning, silent speech was used to avoid movement artefacts.

First, we confirmed that the tasks had been performed correctly. Figure 2A-B shows the mean durations and standard error of the mean (SEM) for all subjects of the individual temporal intervals produced in the isochronous (Fig. 2A) and sequential (Fig. 2B) conditions. Data from the whole scanning is included. To evaluate the accuracy of the performance, we calculated the relative error of each produced temporal interval. For the isochronous conditions, the relative error was calculated as produced interval duration divided by ideal interval duration. For the sequential conditions, we also analysed the consistency of relative errors in the production of different intervals of the sequence, across effectors (see below). For this purpose, it was important to remove effects of small drifts in overall tempo, by normalizing each produced interval duration to the total cycle duration of the whole produced sequence. The relative error $(\delta \mathrm{d})$ was thus calculated as:

$$
\delta d=(d / c) /\left(d_{\mathrm{i}} / c_{\mathrm{i}}\right)
$$

where $d$ is interval duration, $c$ is total cycle duration of the rhythmic sequence, $d_{\mathrm{i}}$ is ideal interval duration, and $c_{\mathrm{i}}$ is ideal cycle duration.

The mean relative error, averaged over all produced intervals by all subjects during the scanning, was $<5.0 \%$ for all conditions (Fig. 2A and B). The temporal structure of the different sequences was thus reproduced with high accuracy. No significant difference was found in
B
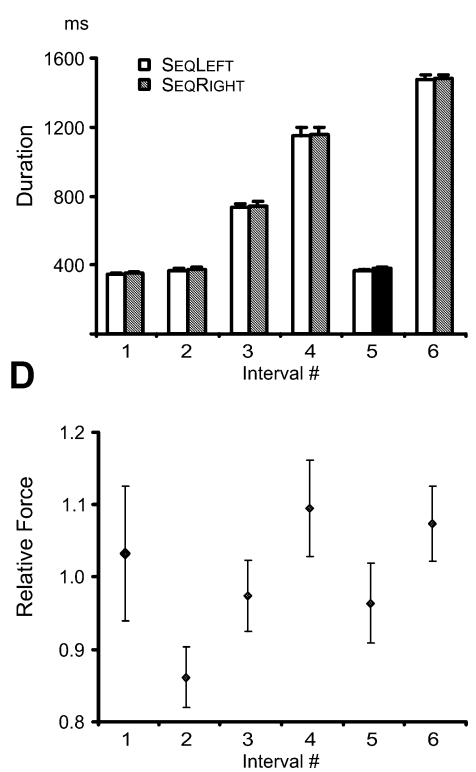

FIG. 2. Timing (A-B) and force (C-D) data from the manual tasks. All graphs show mean values from all subjects and all sessions during the fMRI scans. Error bars in A-C indicate SEM (standard error of the mean), in D the standard deviation. (A) Mean interval between taps in the isochronous tasks. (B) Mean durations of the six different temporal intervals in the sequential tasks. (C) Mean tapping force for all manual tasks. All six intervals were pooled together for the two sequential tasks. (D) Mean relative force for the different intervals in the sequential tasks. The mean tapping forces produced at each interval were first normalized to the mean tapping force of the whole sequence, for each subject. Thereafter, mean relative forces for each interval were averaged across all subjects. 
mean relative error between conditions (single factor ANOVA; main effect of condition on relative error; d.f. $=3 ; F=0.074 ; P=0.97$ ). Likewise, no significant difference in mean tap force was found between the different conditions (Fig. 2C; single factor ANOVA, main effect of condition on force; d.f. $=3 ; F=0.11 ; P=0.95)$. Importantly, as the number of movements was matched between the conditions, we assume that the total motor output was approximately the same in the different conditions.

Secondly, we investigated if any significant improvement of performance took place during the scanning sessions. For this purpose a comparison of performance in the beginning and the end of the scanning was made. The relative error and SD of the temporal intervals produced in the first and last epoch of the experiment were calculated for each condition and subject. Pooling together data from all subjects, a significant change in relative error was found for two conditions, ISOLEFT (decrease of $4.6 \%$; $P<0.004$; paired $t$-test) and RIGHTSEQ (decrease of $0.7 \% ; P<0.005$ ). No significant difference in SD was found for any condition. All tasks were thus well learned and performed correctly without any major changes of behaviour during the scanning.

\section{Correlations between temporal patterns produced with different} effectors

Although the subjects performed the tasks correctly, individual deviations from the ideal temporal pattern were unavoidable. These deviations were useful when addressing the question of whether the same internal representation of the temporal pattern is used to time the movements, independently of which effector is used. If this were the case, one would predict that each subject's characteristic deviations from the ideal temporal pattern would be highly similar during performance of SEQLEFT, SEQRIGHT, and SEQSPEECH.

To investigate this, the detailed temporal structure of the performance of SEQLEFT and SEQRIGHT during scanning was analysed. As SEQSPEECH was performed as speech imagery during scanning, the last 20 performances of the training session, where overt speech was used, were analysed instead. While the detailed characteristics of the temporal structure varied substantially between subjects, individual subjects showed a remarkable consistency in the tasks. Figure $3 \mathrm{~A}$ shows the mean relative error of all six intervals in the sequence, during performance of SEQLEFT, SEQRIGHT, and SEQSPEECH by one subject. An almost perfect overlap in mean duration of all intervals can be observed.

When pooling data from all subjects, high positive within-subject correlations were found for the mean relative error of the same temporal interval of the sequence during performance of the three different tasks. Each dot in the scatter plots of Fig. 3B-D represents the mean relative error of one particular temporal interval, as performed by the same subject in two different tasks. A remarkably strong correlation was found between SEQLEFT and SEQRIGHT $\left(P<5 \times 10^{-19} ; r=0.95\right.$; Pearson Product-Moment Correlation; Fig. 3B). Significant strong correlations were also found between SEQLEFT and SEQSPEECH $(P<0.00007 ; r=0.61$; Fig. $3 C)$ and
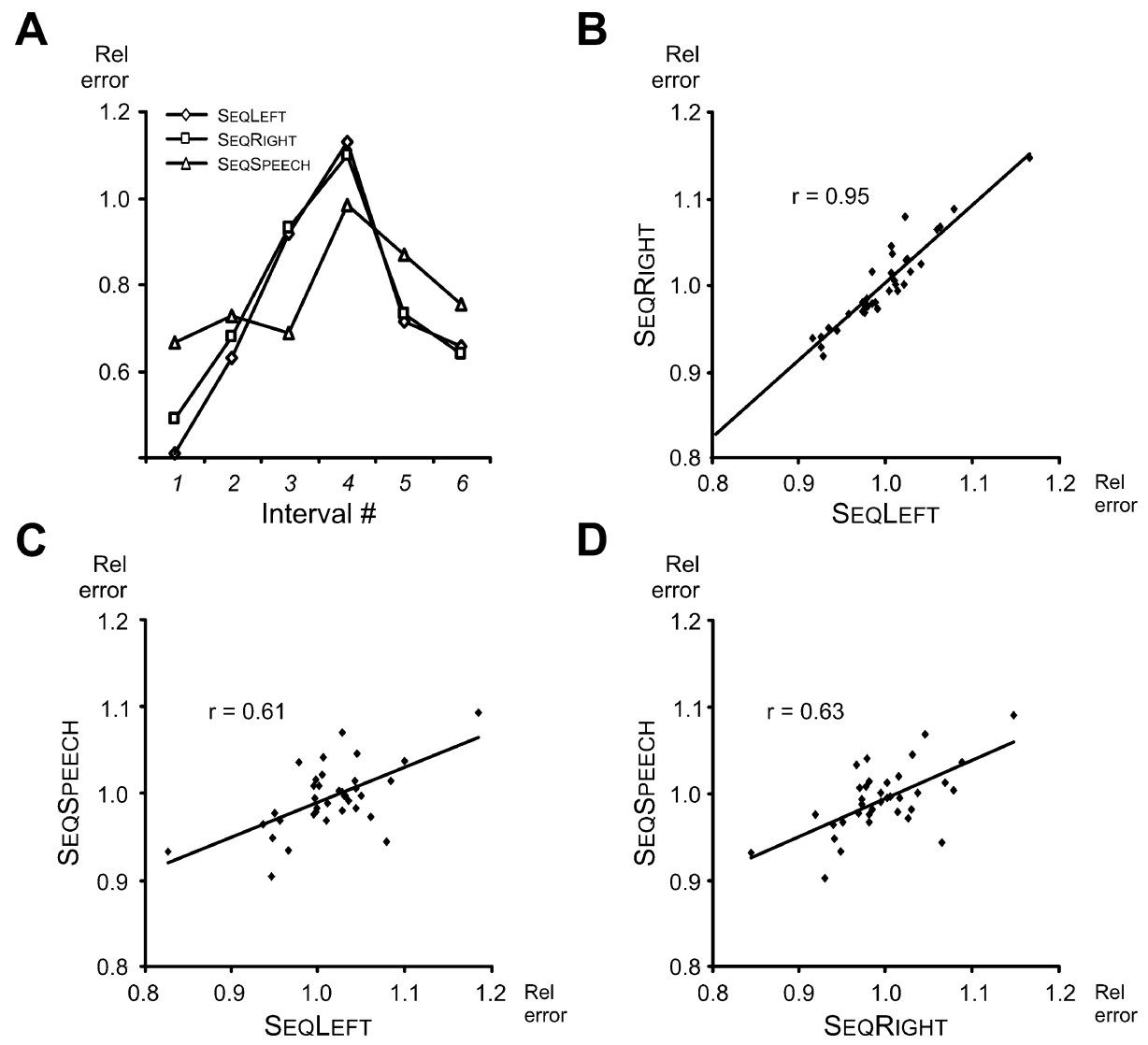

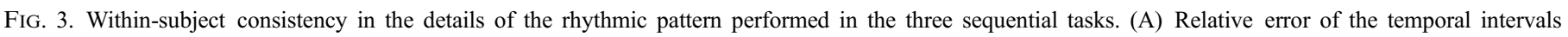

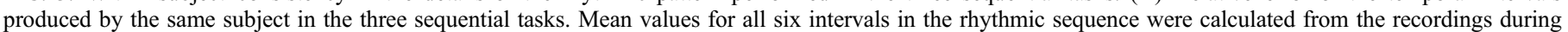

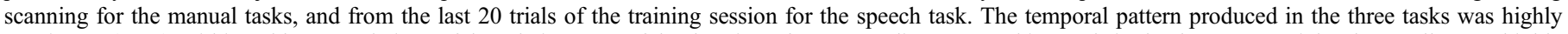

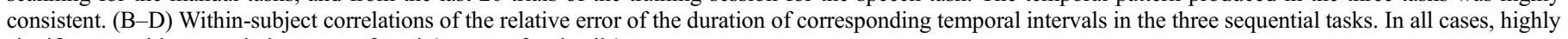
significant positive correlations were found (see text for details). 
between SEQRIGHT and SEQSPEECH $(P<0.0003 ; r=0.63$; Fig. 3D). Notably, the correlation between the two manual tasks SEQLEFT and SEQRIGHT was significantly stronger than the correlation between either manual task and SEQSPEECH (SEQLEFT vs. SEQSPEECH, $P<0.00001$; SEQRIGHT vs. SEQSPEECH, $P<0.00001$; Difference Test for Pearson R's). No significant difference was found between the strengths of the correlations SEQLEFT vs. SEQSPEECH and SEQRIGHT vs. SEQSPEECH $(P=0.90)$. The results of this correlational analysis were corroborated by a multifactor ANOVA analysis, using relative error as dependent variable and subject, effector, and temporal interval number (1-6) as three factors. There was a significant effect on relative error of temporal interval number (d.f. $=5 ; F=10.37 ; P<0.00001$ ), while effector (d.f. $=2 ; \quad F=0.12 ; P=0.89)$ and subject (d.f. $=5$; $F=0.52 ; P=0.76)$ had no effects.

A possible confound is that these correlations were inflated by a nonspecific tendency for larger relative errors for longer intervals. We therefore performed the same correlational analysis, pooling only the three short intervals with a target duration of $375 \mathrm{~ms}$ (i.e. intervals number 1, 2, and 5 of the sequence; see Fig. 1). Again, significant correlations of similar magnitudes were found between all conditions: SEQLEFT $\times$ SEQRIGHT, $P<4 \times 10^{-9}, \quad r=0.94 ; \quad$ SEQLEFT $\times$ SEQSPEECH,$\quad P<0.006 ; \quad r=0.62 ; \quad$ and SEQRIGHT $\times$ SEQSPEECH, $P<0.0009 ; \quad r=0.62$. A multifactor ANOVA analysis showed a significant effect on relative error of temporal interval number (d.f. $=2 ; F=31.22 ; P<4 \times 10^{-9}$ ) and subject (d.f. $=5$; $F=6.35 ; P<0.0002$ ), while effector (d.f. $=2 ; F=0.81 ; P=0.45$ ) had no effect. The results of the correlational analysis of all intervals were thus corroborated.

\section{Tap force and temporal structure}

As our primary interest was timing of isochronous movements and rhythmic sequences, it was important to investigate the tap forces used for the different intervals of the sequence, in order to see if subjects were also producing independent force sequences. For the rhythmic sequence conditions, this analysis revealed clear differences in mean the tap force used for the six different intervals of the sequence (Fig. 2D; single-factor ANOVA; main effect of sequence interval on force; d.f. $=5 ; F=23.08 ; P<0.00001)$.

Metrical sequences of the type used in this study are generally thought to be represented in a hierarchical fashion, i.e. subdivided (chunked) into metrical units (corresponding to musical 'bars') of equal duration, which in turn are subdivided into shorter temporal intervals (see Discussion). The present sequence can be divided into three such metrical units, each 1.5-s long (see vertical arrows in Fig. 1). Notably, intervals 1, 4, and 6 were produced with higher mean force (Fig. 2D). The onsets of these intervals occur at the starting points of the metrical units of the sequence $(t=0 \mathrm{~s}, 1.5 \mathrm{~s}$, and $3.0 \mathrm{~s}$; cf. Figure 1). A multiple regression analysis was performed as follows:

$$
T=b_{0}+b_{1} D+b_{2} M
$$

where $b_{0}, b_{1}$ and $b_{2}$ are regression coefficients; $T$ is mean tap force; $D$ is mean interval duration; and $M$ is a discrete variable that assumed the value 2 for intervals 1,4 , and 6 , that occurred at onsets of metrical units, and the value 1 for intervals 2,3 , and 5 , that occurred within metrical units. A strong $(R=0.72)$ and highly significant $\left(P<0.00001 ; F_{2,69}=37.4\right)$ relation was found. The beta coefficients corresponding to $b_{1}$ and $b_{2}$ were both significantly different from zero: $\beta_{1}=0.28\left(P<0.007 ; t_{69}=2.79\right)$ and $\beta_{2}=0.52(P<0.00001 ;$ $\left.t_{69}=5.17\right)$, respectively. The tap forces were thus predominantly determined by the metrical structure of the sequence $\left(\beta_{2}\right)$, where stronger taps ('accents') mark onsets of new metrical units, and to a lesser extent by the durations of the individual intervals $\left(\beta_{1}\right)$, where longer intervals tend to be preceded by stronger taps.

\section{Functional MRI data}

\section{Effector-independent control of rhythmic sequences}

The left column of Table 1 (Conjunction SEQ - REST) and the left part of Fig. 4 summarize the activations seen in the conjunction analysis of the three contrasts SEQRIGHT - REST, SEQLEFT - REST, and SEQSPEECH - REST.

On the medial wall of the frontal lobe a large cluster of active voxels was found in the left SMA, extending ventrally into the caudal

TABLE 1. Brain regions with significant* increase in BOLD contrast signal in the two conjunction analyses

\begin{tabular}{|c|c|c|c|c|c|c|}
\hline Brain region & Side & $x$ & $y$ & $z$ & $t_{\min }^{\dagger}$ & Single $\mathrm{Ss}^{\star}$ \\
\hline \multicolumn{7}{|l|}{ Conjunction SEQ - REST } \\
\hline SMA/preSMA/CMAc & $\mathrm{L}$ & -8 & -4 & 56 & 18.20 & AAAAACD \\
\hline Precentral g. & $\mathrm{L}$ & -60 & -4 & 36 & 7.39 & AAABCDE \\
\hline (PMV/PMD) & & -56 & -12 & 44 & 8.09 & AABCDDE \\
\hline Ant STG - BA $6 / 44^{\S}$ & $\mathrm{L}$ & -56 & 4 & 0 & 7.42 & $\mathrm{ABBCCCD}$ \\
\hline Post STG & $\mathrm{L}$ & -52 & -44 & 16 & 9.56 & AABDDEE \\
\hline Globus pallidus & $\mathrm{L}$ & -24 & -4 & -8 & 5.02 & BBDDEEE \\
\hline $\begin{array}{l}\text { Cerebellum, vermis } \\
\text { lobule } \mathrm{V} / \mathrm{VI}^{\dagger \dagger}\end{array}$ & $\mathrm{L}$ & -8 & -64 & -24 & 11.37 & AABCDEE \\
\hline Cerebellum, hemisph. & $\mathrm{L}$ & -32 & -64 & -32 & 9.67 & AAAABDD \\
\hline lobule $\mathrm{VI}^{\dagger \dagger}$ & $\mathrm{R}$ & 32 & -60 & -32 & 10.99 & AAADDDD \\
\hline \multirow[t]{2}{*}{ Thalamus } & $\mathrm{L}$ & -16 & -12 & 16 & 5.46 & ADDDEEE \\
\hline & $\mathrm{R}$ & 12 & -12 & 16 & 5.89 & ABDDEEE \\
\hline \multicolumn{7}{|c|}{ Conjunction ISO - REST } \\
\hline SMA/preSMA/CMAc & $\mathrm{L}$ & -8 & -4 & 56 & 15.48 & AACCCDE \\
\hline $\begin{array}{l}\text { Precentral g. } \\
\text { (PMV/PMD) }\end{array}$ & $\mathrm{L}$ & -44 & -12 & 52 & 5.53 & CDDDDEE \\
\hline Ant STG - BA $6 / 44^{\S}$ & $\mathrm{L}$ & -56 & 0 & -4 & 7.57 & CCDDDDE \\
\hline Post STG & $\mathrm{L}$ & -56 & -28 & 16 & 5.22 & BCDDDDE \\
\hline Cerebellum, vermis & $\mathrm{L}$ & -8 & -64 & -20 & 6.50 & AABCCDD \\
\hline lobule $\mathrm{V} / \mathrm{VI}^{\dagger \dagger}$ & $\mathrm{R}$ & 8 & -72 & -20 & 5.25 & BCDDDDD \\
\hline Cerebellum, hemisph. & $\mathrm{L}$ & -32 & -64 & -28 & 5.09 & ADDDDDE \\
\hline lobule $\mathrm{VI}^{\dagger \dagger}$ & $\mathrm{R}$ & 32 & -60 & -32 & 7.35 & BBCDDDD \\
\hline \multicolumn{7}{|l|}{ Conjunction SEQ - ISO } \\
\hline SMA/preSMA/CMAc & $\mathrm{L}$ & -8 & 0 & 56 & $2.93 * *$ & ABCDDDD \\
\hline Post STG & $\mathrm{L}$ & -52 & -40 & 20 & $1.97 * *$ & ABCCDDE \\
\hline $\begin{array}{l}\text { Cerebellum, vermis } \\
\text { lobule } \mathrm{V} / \mathrm{VI}^{\dagger \dagger}\end{array}$ & $\mathrm{R}$ & 4 & -64 & -16 & $4.00 * *$ & ABCCCDE \\
\hline Cerebellum, hemisph. & $\mathrm{L}$ & -36 & -38 & -32 & $3.46^{* *}$ & CDDDEEE \\
\hline \multirow{2}{*}{ lobule $\mathrm{VI}^{\dagger \dagger}$} & $\mathrm{R}$ & 28 & -44 & -40 & 4.56 & BDDDDDE \\
\hline & & 40 & -56 & -32 & 5.99 & ABCDDDE \\
\hline
\end{tabular}

Results from the three conjunction analyses SEQ - REST (SEQRIGHT - REST, SEQLEFT - REST, SEQSPEECH - REST), ISO - REST (ISORIGHT - REST, ISOLEFT - REST, ISOSPEECH - REST) and SEQ - ISO (SEQRIGHT - ISORIGHT, SEQLEFT - ISOLEFT, SEQSPEECH - ISOSPEECH) are shown in separate columns. BA, Brodmann area; $* P<0.05$, corrected for multiple comparisons. $x, y$, and $z$ give the coordinates in standard space of the activity peak. ${ }^{\dagger}$ The minimum $t$-scores of each peak voxel across the contrasts in the conjunction. ${ }^{+}$The level of activity in the individual participants, for each active region in the group analysis. Each subject is allocated a letter from $\mathrm{A}$ to $\mathrm{E}$ to indicate significance level, as follows: A, $P \leq 0.05$ corrected; B, $P \leq 0.001$ uncorrected; C, $0.001<P \leq 0.01$ uncorrected; D, $0.01<P \leq 0.05$ uncorrected; E, not significant at $P<0.05$ uncorrected. ${ }^{\S}$ In both conjunctions, the clusters extended into the anterior portion of the STG and into BA areas $6 / 44$ of the adjacent frontal lobe. ${ }^{\dagger \dagger}$ Cerebellar lobules are given after Schmahmann et al. (2000). ${ }^{*} P<0.05$, after a small volume correction for multiple comparisons within a spherical region of interest with radius $10 \mathrm{~mm}$, and centre in the peak coordinate of the cluster in the corresponding region, seen in Bengtsson et al. (2004a). 


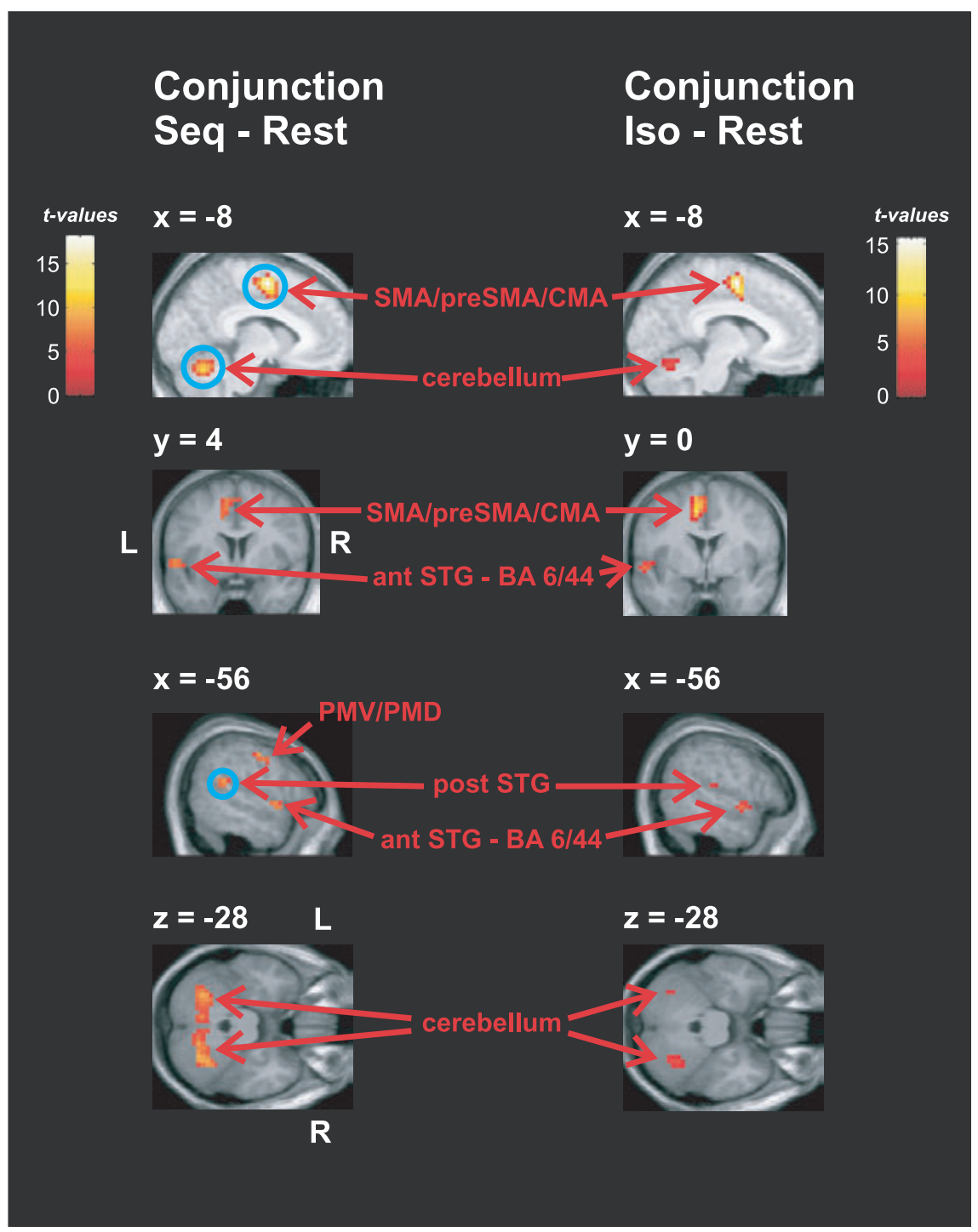

FIG. 4. Activated brain regions in the two conjunction analyses SEQ-REST and ISO-REST. Activity maps of brain regions with significantly increased BOLD contrast signals are shown $(P<0.05$ corrected; the colour scale indicate the $t$-values). The coordinates for the displayed slices are indicated. $\mathrm{R}$ and $\mathrm{L}$ denote the right and left hemispheres, respectively. Blue circles in the left column indicate regions that were significantly active in a SEQ-ISO conjunction, after small volume correction.

cingulate motor area (CMAc) and rostrally to the SMA/preSMA border $(y=0)$. On the lateral surface of the frontal lobe, a cluster of activity was found in the left precentral gyrus, in the region of the dorsal (PMD) and ventral (PMV) premotor cortices. Two activated regions were found in the temporal lobe, also on the left side. The first cluster was located in the anterior superior temporal gyrus (STG) and Brodmann areas $6 / 44$ of the neighbouring frontal lobe. The other cluster was situated in the posterior STG, close to the temporo-parietal junction. Subcortical activations were found in the left globus pallidus, in the left lobule V/VI of the vermis, bilaterally in lobule VI of the cerebellar hemispheres, and bilaterally in the ventrolateral thalamus.

\section{Effector-independent control of isochronous movements}

Brain regions activated in the conjunction of ISORIGHT - REST, ISOLEFT - REST and ISOSPEECH - REST (Conjunction ISO REST) are shown in the middle column of Table 1 and the right part of Fig. 4. In general, the pattern of brain activity was highly similar to that seen in the conjunction analysis of the rhythmic sequence conditions vs. rest. Cortical activity was found in the same regions of the left frontal and temporal lobes as in the previous conjunction. The two frontal clusters were localized in the SMA, extending into CMAc and rostrally to the preSMA border, and in the PMD, extending into the PMV. Activity was found in the anterior STG, area 6/44 of the inferior frontal cortex, and in the posterior STG. Subcortical activations were restricted to the cerebellum: lobule V/VI bilaterally in the vermis, and lobule VI bilaterally in the hemispheres.

\section{Differences between effector-independent control of rhythmic sequences and isochronous movements}

Finally, we investigated which brain regions showed a significantly stronger activation during rhythmic sequence performance than during isochronous performance, independently of effector. We thus examined the conjunction of the contrasts SEQRIGHT - ISORIGHT, SEQLEFT - ISOLEFT and SEQSPEECH - ISOSPEECH. As summarized in the right column of Table 1, in this case we found activity in a subset of the regions activated in the other two conjunctions, 
which are indicated with blue circles in the left column of Fig. 4 $(P<0.05$, based on a small volume correction). Cortical activity was found in the left mesial premotor areas (SMA/preSMA extending into the CMAc) and in the left posterior part of the STG close to the temporo-parietal junction. Subcortical activations were found in the right vermis (lobule V/VI), and bilaterally in lobule VI of the cerebellar hemispheres. No activity was found in the basal ganglia, or anywhere else in the brain.

\section{Discussion}

A fundamental question when investigating the neural processing of temporal events is whether the brain utilizes a central process that is recruited when explicit timing is required or, whether temporal information is computed locally within the neural mechanisms required for a particular task (Ivry \& Spencer, 2004; Buhusi \& Meck, 2005). In the present study we have investigated the neural processing of rhythmic sequences. Clearly, several processes are involved here; the intention to produce a rhythm must be present, as well as the decision to do so. Moreover, the timing of intervals, linking these together in a sequence, and maintaining this sequence in short-term memory are processes involved. Here, we have not addressed the question of which brain areas house these various subprocesses, or whether there are one or several timers computing the intervals. Our focus has been on whether there exists a central network of brain areas for rhythm production with different body parts. Indeed, our behavioural data strongly suggests that this is the case. In addition, the set of brain areas we find active in rhythm production invariant of effector, is commonly found active in brain imaging studies on temporal production (Rao et al., 1997; Bengtsson et al., 2004a), as well as perception (Coull et al., 2004). Thus, our results strongly favour the notion that a central network is involved in rhythm processing, independently of the used effector.

\section{Abstract representations of temporal patterns in motor timing - behavioural evidence}

The first indications of effector-independent mechanisms in temporal control came from studies showing positive within-subject correlations for the maximum rate (Keele \& Hawkins, 1982) and temporal variability (Franz et al., 1992; Robertson et al., 1999) of isochronous movements performed with different effectors. Interestingly, later studies have shown that such correlations in timing variability are seen between rhythmic tasks involving discrete movements (Zelaznik et al., 2002), such as tapping, but not between continuous and discrete motor tasks (Robertson et al., 1999; Zelaznik et al., 2002). While these findings suggest that there is a general ability for voluntary timing of discrete movements, they do not provide strong evidence for the stronger hypothesis that common representations of temporal patterns are used to time movements with different effectors. Here, we examine the details of the temporal pattern of a rhythmic sequence, produced with different effectors. The observed intrasubject correlations in the entire rhythmic sequence, as well as in the separate analysis of the intervals with short target duration, provide evidence that a common representation was indeed used to time the movements in all three tasks. Furthermore, this is in agreement with previous findings of temporal consistency in different spatiotemporal sequences performed with the same effector (Ullén \& Bengtsson, 2003). It appears therefore that in voluntarily timed tasks the brain can represent temporal patterns in the abstract, i.e. independently both of the effector and the spatial structure of the movements. While all tasks showed a high degree of correlation, this was higher between the two manual tasks, than between each manual task and speech. This may reflect the higher degree of similarity of the motor aspects of the manual tasks; the two manual tasks only differ in laterality, while nonhomologous muscle groups have to be activated for finger tapping and speech.

In the manual tasks, the mean tap force used differed systematically in different taps of the sequence (see Fig. 2D). These tap force variations essentially reflected how the temporal sequence was organized metrically; stronger taps ('accents') occurred at the beginning of metrical units. Such accentuation of the first element of metrical units has been demonstrated consistently in both production and perception (Essens, 1995; Semjen \& Vos, 2002). Importantly, we found no evidence that independent sequences of tap forces were produced. An accentuation of the beginning of metrical units thus rather appears to be an integral feature of rhythm production.

\section{The fMRI experiment: methodological considerations}

Although rhythmic sequence performance is a more complex task than isochronous performance, the employed rhythmic sequences were short and simple, and had been trained to the extent that no significant differences in temporal accuracy were found between the sequential and isochronous tasks during scanning. The brain activity found enhanced for sequential rhythm production, in comparison to isochronous, is thus due to the increased demand of timing several different intervals, as well as ordering these in a sequential order. Importantly, the preSMA/SMA, the lateral premotor cortex, the superior temporal cortex have earlier been shown to be specifically involved in temporal sequence control, in a study where temporal and ordinal sequences of matched complexity were contrasted (Bengtsson et al., 2004a).

We assume that the contrasts between rhythmic sequence performance and isochronous performance essentially reveal brain activity related to effector-independent aspects of the production of a temporal pattern. However, differentiating between effector-independent areas and separate, but closely adjacent, effector-dependent areas is obviously not feasible with the present technique. Similarly, the current spatial resolution prevents us from making a more fine-grained analysis of whether subregions within the same brain region are specifically involved in the control of rhythmic sequences or isochrony. However, our findings of activity in the SMA and the posterior parts of the lateral cerebellum in the conjunction analyses, are consistent with previous human imaging studies, finding indications of effector-independent activity in these areas (Rijntjes et al., 1999; Ehrsson et al., 2000). Electrical stimulation studies in human (Fried et al., 1991; Kurata, 1992; Hanakawa et al., 2001) and monkey (Mitz \& Wise, 1987) have also indicated that the somatotopical organization of the human SMA includes regions with overlapping representations of different body parts.

\section{Brain regions involved in effector-independent temporal control}

Increased activity related to the effector-independent control of a rhythmic sequence was found in areas that have earlier been recognized as important for motor timing. Isochronous movements require the timekeeping of a regular beat; rhythmic sequence performance in addition requires the representation of a temporal pattern of different intervals. Notably, our data suggests that effector-independent aspects of these two functions isochronous and rhythm patterns are handled by the same set of brain regions, with some of the regions being of particular importance for the organization of a rhythmic sequence. 
In this context, it is important to distinguish between two types of ordinal information. On the one hand, there is the order of the temporal intervals of a rhythmic sequence. The present results suggest that this control is an integral part of the timekeeping system of the brain. On the other hand, there is the serial order of the individual acts comprising a movement sequence. Earlier work has shown that this aspect is controlled by brain regions different from those controlling the temporal structure of the sequence (Schubotz \& von Cramon, 2001; Bengtsson et al., 2004a). Specifically, it has been shown that the border region of SMA and preSMA shows responses that reflect the control of temporal structures, over and above responses that are associated with ordinal sequence control (Bengtsson et al., 2004a), which is known to activate SMA in humans (Roland et al., 1980; Sadato et al., 1996; Boecker et al., 1998; Schubotz \& von Cramon, 2001; Bengtsson et al., 2004a) and monkeys (Mushiaka et al., 1991; Tanji \& Shima, 1994; Shima \& Tanji, 2000); reviewed in Tanji (2001).

\section{The roles of the mesial premotor areas for effector-independent temporal control}

In all conjunction analyses in the present study, a cluster of activity was found in the left SMA extending rostrally into to the caudal part of the preSMA, and ventrally into the CMAc. This suggests a central role for these mesial premotor areas in effector-independent voluntary motor timing. A large number of studies have consistently demonstrated activity of the SMA proper and/or the preSMA in a variety of tasks that require processing of temporal patterns; reviewed in, e.g. Macar et al. (2002) and Ivry \& Spencer (2004).

Pioneering experiments, using auditory stimuli, suggested that self-paced isochronous tapping activates a medial premotor circuit, including the SMA, the basal ganglia and the thalamus, stronger than does tapping in synchrony with auditory stimuli (Rao et al., 1997). SMA activity has also been seen during rhythmic sequence reproduction (Penhune et al., 1998; Schubotz \& von Cramon, 2001), rhythmic sequence learning (Ramnani \& Passingham, 2001; Sakai et al., 2002), performance of well-learned rhythmic sequences (Bengtsson et al., 2004a; Lewis et al., 2004), and production of discrete temporal intervals with a particular duration (Macar et al., 1999). The same region is active in sensory tasks requiring processing of temporal information (Macar et al., 2002), such as encoding and discrimination of rhythmic sequences (Schubotz et al., 2000; Schubotz \& von Cramon, 2001), passive perception of rhythmic sequences without any additional task (Bengtsson et al., 2004b), and discrimination of discrete temporal intervals (Macar et al., 1999). The present findings are important as they, together with previous work (Bengtsson et al., 2004a), clearly demonstrate that the activity in the border zone between SMA and preSMA during temporal sequence performance occurs independently of both the effector and the spatial structure of the movements. This, and the fact that the SMA/preSMA region is active in such a large variety tasks involving voluntary timing, suggests that neural activity in this region can form abstract, amodal representations of temporal patterns, which can be used for perceptual tasks, as well as during learning and performance.

To our knowledge, no neurophysiological studies in monkeys have been conducted to investigate rhythmic sequence production and, indeed, voluntary production of temporal patterns may be a specifically human capacity (Merker, 2000). Of interest in relation to the present study, however, are the demonstrations that neuronal activity in the mesial premotor cortex of the macaque monkey reflects the ordinal structure of sequences of both saccadic eye movements (Isoda
\& Tanji, 2004) and hand movements (Tanji \& Shima, 1994; Shima \& Tanji, 2000), suggesting an involvement in effector-independent aspects of the sequence control.

It appears difficult to draw definitive conclusions with regard to the specific roles of the different medial wall motor areas for motor timing at present. Recent experiments using TMS to disrupt preSMA activity during finger sequences (Kennerley et al., 2004) suggest a specific role for this region in sequence initiation and the hierarchical organization of the sequence (chunking). It remains to be investigated if the preSMA plays similar roles for the hierarchical organization of longer rhythmic sequences. The activity in CMAc observed in the present study is consistent with a recent observation of activity in this region during rhythmic sequence performance (Lewis et al., 2004).

\section{The STG and auditory-motor integration}

Activity in the STG is consistently seen in voluntary timing tasks involving auditory stimuli. Among motor tasks can be mentioned stimulus synchronized and self-paced finger tapping, both isochronous (Rao et al., 1997; Jäncke et al., 2000; Lutz et al., 2000; Jantzen et al., 2004, 2005) and using rhythmic sequences (Lewis et al., 2004), as well as rhythmic sequence reproduction (Penhune et al., 1998) and performance of spatiotemporal sequences from memory (Bengtsson et al., 2004a). Deficits in perception of temporal patterns have consistently been demonstrated after lesions to the STG (Liégeois-Chauvel et al., 1998; Ayotte et al., 2000; Wilson et al., 2002). Involvement of auditory areas in the superior temporal cortices may reflect that the sequences were learned from auditory stimuli, or that an auditory metronome was used to set the tempo. In the synchronization/continuation paradigm, superior temporal activity is seen with isochronous auditory stimuli, but not with visual stimuli (Jäncke et al., 2000; Jantzen et al., 2005). Here, we found activity in two locations in the left STG. The activation in the anterior pole is consistent with lesion studies demonstrating the importance of this region for rhythmic processing (Liégeois-Chauvel et al., 1998). The cluster in the posterior dorsal STG is of particular interest. This region has recently been suggested to play a key role for auditory-motor integration during both speech and nonlinguistic tasks (Hickok et al., 2003). According to this hypothesis the left posterior dorsal STG is part of a dorsal auditory stream that connects superior temporal, inferior frontal and inferior parietal cortical regions in a network for auditory-motor control (Hickok \& Poeppel, 2000). The present results suggest this network is involved in the performance from memory of rhythmic patterns that were learned from auditory stimuli. To examine the possibility that the participants engage in covert humming during the experiment, the BOLD-signal in the M1 mouth area [peak voxel $x=52, y=-8, z=36$ taken from Lotze et al. (2000)] was examined (not shown). Importantly, a deactivation of the region relative to REST was found in both manual conditions, which does not support that subjects used covert humming. The role of the STG in rhythm production should be further addressed in experiments specifically designed for the matter, however.

\section{Subcortical regions and timing}

The extensive activations seen in cerebellum corroborate the key role of cerebellum in temporal control, as has been demonstrated in a large body of studies (reviewed in, e.g. Ivry et al., 2002; Ivry \& 
Spencer, 2004). A persistent question in the timing literature has been whether cerebellar activity in temporal tasks reflects direct involvement in the processing of temporal information, or whether it is rather related to more general sensori-motor functions (Rao et al., 2001). In this regard, it is noteworthy that cerebellar activity was observed in the present study although the silent speech task did not include overt movements, which is consistent with a specific role for cerebellum in timing.

Several studies have shown that the basal ganglia play important roles for timing (Rao et al., 1997; Harrington et al., 1998; Lewis et al., 2004); for a recent review see, e.g. Ivry \& Spencer (2004). Whether the basal ganglia play specific roles for the production of rhythmic sequences of several intervals is less clear. Lewis et al. (2004) found a parametric increase of activity in the putamen as a function of rhythmic sequence length, during the initiation phase of rhythm performance. However, other previous studies have failed to detect basal ganglia activity related to the learning of a rhythmic sequence (Ramnani \& Passingham, 2001; Sakai et al., 2002). Basal ganglia involvement in rhythm control may depend on stage of learning. Penhune \& Doyon (2002) found increased basal ganglia activity during delayed recall of previously learned rhythmic sequences, and Puttemans et al. (2005) similarly found increasing basal ganglia activity during prolonged ( 2 weeks) intense practising of a rhythmic bimanual coordination task. Long-lasting retention of motor sequences in general has been proposed to involve the basal ganglia (Doyon et al., 2002; Ungerleider et al., 2002; Doyon \& Benali, 2005) as well as the cerebellum (Hikosaka et al., 2002; Puttemans et al., 2005).

Here, we did not detect an effector-independent activation of the basal ganglia in the SEQ-ISO conjunction. Overall, the data thus remains inconclusive with regard to the specific functions of the basal ganglia for rhythmic sequence production.

\section{General conclusions}

In summary, we provide evidence that humans can form effectorindependent representations of temporal patterns. A set of motor areas and temporal and subcortical brain regions are involved in this type of control, where the SMA/preSMA/CMAc, the STG, and the cerebellum appear particularly important for the handling of rhythmic sequences of several intervals. They constitute a neural substrate for the everyday experience that a well-learned rhythm can as easily be produced with the hand, with the foot or by singing. These findings suggest that production of rhythms from memory may rely on rehearsal of auditory memories using the same neural circuitry for auditory-motor integration as subvocal speech, and support the view that the medial premotor areas are critically involved in timing and effector-independent sequence control.

\section{Acknowledgements}

This work was supported by the Swedish Research Council, the Jeansson Foundations, Sällskapet Barnavård, Karolinska Institutet's Research Funds, Linnea \& Josef Carlssons stiftelse, and the Freemasons in Stockholm Foundation for Children's Welfare.

\section{Abbreviations}

BOLD, blood oxygenation level dependent; CMA, cingulate motor area; CMAc, caudal cingulate motor area; PMD, dorsal premotor cortex; PMV, ventral premotor cortex; preSMA, presupplementary motor area; SMA, supplementary motor area; STG, superior temporal gyrus.

\section{References}

Ayotte, J., Peretz, I., Rousseau, I., Bard, C. \& Bojanowski, M. (2000) Patterns of music agnosia associated with middle cerebral artery infarcts. Brain, 123, 1926-1938.

Bengtsson, S., Ehrsson, H.H., Forssberg, H. \& Ullén, F. (2004a) Dissociating brain regions controlling the temporal and ordinal structure of learned movement sequences. Eur. J. Neurosci., 19, 2591-2602.

Bengtsson, S.L., Hashimoto, T., Tomonori, K., Ullén, F., Naito, E., Forssberg, H. \& Sadato, N. (2004b) Perception of temporal regularity in auditory stimuli. Neuroimage, 22, Suppl. 1, e915. [Human Brain Mapping 2004 conference abstract].

Boecker, H., Dagher, A., Ceballos-Baumann, A.O., Passingham, R.E., Samuel, M., Friston, K.J., Poline, J., Dettmers, C., Conrad, B. \& Brooks, D.J. (1998) Role of the human rostral supplementary motor area and the basal ganglia in the motor sequence control: investigations with 15H20 PET. J. Neurophysiol., 79, 1070-1080.

Buhusi, C.V. \& Meck, W.H. (2005) What makes us tick? Functional and neural mechanisms of interval timing. Nature Rev. Neurosci., 6, 755-765.

Coull, J.T., Vidal, F., Nazarian, B. \& Macar, F. (2004) Functional anatomy of the attentional modulation of time estimation. Science, 303, 1506-1508.

Doyon, J. \& Benali, H. (2005) Reorganization and plasticity in the adult brain during learning of motor skills. Curr. Opin. Neurobiol., 15, 161-167.

Doyon, J., Song, A.W., Karni, A., Lalonde, F., Adams, M.M. \& Ungerleider, L.G. (2002) Experience-dependent changes in cerebellar contributions to motor sequence learning. Proc. Natl Acad. Sci. USA, 99, 1017-1022.

Duvernoy, H.M. (2000) The Human Brain: Surface, Blood Supply and ThreeDimensional Sectional Anatomy. Springer, Wien.

Ehrsson, H.H., Naito, E., Geyer, S., Amunts, K., Zilles, K., Forssberg, H. \& Roland, P.E. (2000) Simultaneous movements of upper and lower limbs are coordinated by motor representations that are shared by both limbs: a PET study. Eur. J. Neurosci., 12, 3385-3398.

Essens, P. (1995) Structuring temporal sequences: comparison of models and factors of complexity. Percept. Psychophys., 57, 519-532.

Franz, E.A., Zelaznik, H.N. \& Smith, A. (1992) Evidence of common timing processes in the control of manual, orofacial, and speech movements. J. Motor Behav., 24, 281-287.

Fried, I., Katz, A., McCarthy, G., Sass, K.J., Williamson, P., Spencer, S.S. \& Spencer, D.D. (1991) Functional organization of human supplementary motor cortex studied by electrical stimulation. J. Neurosci., 11, 3656-3666.

Friston, K.J., Holms, A.P., Worsley, K.J., Poline, J.-B., Frith, C.D. \& Frackowiak, R.S.J. (1995) Statistical parametric maps in functional imaging: a general linear approach. Hum. Brain Mapp., 2, 189-210.

Halsband, U., Ito, N., Tanji, J. \& Freund, H.J. (1993) The role of premotor and the supplementary motor area in the temporal control of movement in man. Brain, 116, 243-266.

Hanakawa, T., Ikeda, A., Sadato, N., Okada, T., Fukuyama, H., Nagamine, T., Honda, M., Sawamoto, N., Yazawa, S., Kunieda, T., Ohara, S., Taki, W., Hashimoto, N., Yonekura, Y., Konishi, J. \& Shibasaki, H. (2001) Functional mapping of human medial frontal areas. The combined use of functional magnetic resonance imaging and cortical stimulation. Exp. Brain Res., 138, 403-409.

Harrington, D.L., Haaland, K.Y. \& Hermanowicz, N. (1998) Temporal processing in the basal ganglia. Neuropsychology, 12, 3-12.

Harrington, D.L., Lee, R.R., Boyd, L.A., Rapcsak, S.Z. \& Knight, R.T. (2004) Does the representation of time depend on the cerebellum? Effect of cerebellar stroke. Brain, 127, 561-574.

Hickok, G., Buchsbaum, B., Humphries, C. \& Muftuler, T. (2003) Auditorymotor interaction revealed by fMRI: speech, music, and working memory in area Spt. J. Cogn. Neurosci., 15, 673-682.

Hickok, G. \& Poeppel, D. (2000) Towards a functional neuroanatomy of speech perception. Trend Cogn. Sci., 4, 131-138.

Hikosaka, O., Nakamura, K., Sakai, K. \& Nakahara, H. (2002) Central mechanisms of motor skill learning. Curr. Opin. Neurobiol., 12, $217-222$.

Isoda, M. \& Tanji, J. (2004) Participation of the primate presupplementary motor area in sequencing multiple saccades. J. Neurophysiol., 92, 653-659.

Ivry, R. \& Keele, S.W. (1989) Timing functions of the cerebellum. J. Cogn. Neurosci., 1, 136-152.

Ivry, R.B., Keele, S.W. \& Diener, H.C. (1988) Dissociation of the lateral and medial cerebellum in movement timing and movement execution. Exp. Brain Res., 73, 167-180.

Ivry, R. \& Spencer, R.M.C. (2004) The neural representation of time. Curr. Opin. Neurobiol., 14, 225-232.

Ivry, R.B., Spencer, R.M., Zelaznik, H.N. \& Diedrichsen, J. (2002) The cerebellum and event timing. Ann. N.Y. Acad. Sci., 978, 302-317. 
Jäncke, L., Loose, R., Lutz, K., Specht, K. \& Shah, N.J. (2000) Cortical activations during paced finger-tapping applying visual and auditory pacing stimuli. Cogn. Brain Res., 10, 51-66.

Jantzen, K.J., Steinberg, F.L. \& Kelso, J.A.S. (2004) Brain networks underlying timing behavior are influenced by prior context. Proc. Natl Acad. Sci., 101, 6815-6820.

Jantzen, K.J., Steinberg, F.L. \& Kelso, J.A.S. (2005) Functional MRI reveals the existence of modality and coordination-dependent timing networks. Neuroimage, 25, 1031-1042.

Keele, S.W. \& Hawkins, H.L. (1982) Explorations of individual differences relevant to high level skill. J. Motor. Behav., 14, 3-23.

Keele, S.W. \& Ivry, R.I. (1987) Modular analysis of timing in motor skill. Psychol. Learning Motiv., 21, 183-228.

Kennerley, S.W., Sakai, K. \& Rushworth, M.F.S. (2004) Organization of action sequences and the role of the pre-SMA. J. Neurophysiol., 91, 978-993.

Kurata, K. (1992) Somatotopy in the human supplementary motor area. TINS, 15, 159-160.

Kwong, K.K., Belliveau, J.W., Chesler, D.A.I.E.G., Weisskoff, R.M., Poncelet, B.P., Kennedy, D.N., Hoppel, B.E., Cohen, M.S. \& Turner, R. (1992) Dynamic magnetic resonance imaging of the human brain activity during primary sensory stimulation. Proc. Natl Acad. Sci. USA, 89, 56755679.

Lashley, K.S. (1967) The problem of serial order in behavior. In Jeffress, L.A., (Ed), Cerebral Mechanisms in Behavior. Hafner Publishing Co., New York, pp. $112-146$.

Lewis, P.A., Wing, A.M., Pope, P.A., Praamstra, P. \& Miall, R.C. (2004) Brain activity correlates differentially with increasing temporal complexity of rhythms during initialisation, synchronisation, and continuation phases of paced finger tapping. Neuropsychologia, 42, 1301-1312.

Liégeois-Chauvel, C., Peretz, I., Babaï, M., Laguitton, V. \& Chauvel, P. (1998) Contribution of different cortical areas in the temporal lobes to music processing. Brain, 121, 1853-1867.

Logothetis, N.K. (2002) The neural basis of the blood-oxygen-level-dependent functional magnetic resonance imaging signal. Philos. Trans. R. Soc. Lond. B Biol. Sci., 357, 1003-1037.

Lotze, M., Erb, M., Flor, H., Huelsmann, E., Godde, B. \& Grodd, W. (2000) fMRI evaluation of somatotopic representation in human primary motor cortex. Neuroimage, 11, 473-481.

Lutz, K., Specht, K., Shah, N.J. \& Jäncke, L. (2000) Tapping movements according to regular and irregular visual timing signals investigated with fMRI. Neuroreport, 11, 1301-1306.

Macar, F., Lejeune, H., Bonnet, M., Ferrara, A., Pouthas, V., Vidal, F. \& Maquet, P. (2002) Activation of the supplementary motor area and of attentional networks during temporal processing. Exp. Brain Res., 142, 475485.

Macar, F., Vidal, F. \& Casini, L. (1999) The supplementary motor area in motor and sensory timing: evidence from slow brain potential changes. Exp. Brain Res., 125, 271-280.

Martin, J.G. (1972) Rhythmic (hierarchical) versus serial structure in speech and other behavior. Psychol. Rev., 79, 487-509.

Merker, B. (2000) Synchronous Chorusing and Human Origins. In Wallin, N.L., Merker, B., \& Brown, S., (Eds), MIT Press, Cambridge, MA, USA, pp. 315-329.

Mitz, A.R. \& Wise, S.P. (1987) The somatotopic organization of the supplementary motor area: intracortical microstimulation mapping. J. Neurosci., 7, 1010-1021.

Mushiaka, H., Inase, M. \& Tanji, J. (1991) Neuronal activity in the primate premotor, supplementary, and precentral motor cortex during visually guided and internally determined sequential movements. J. Neurophysiol., 66, 705718 .

Nichols, T., Brett, M., Andersson, J., Wager, T. \& Poline, J.-B. (2005) Valid conjunction inference with the minimum statistic. Neuroimage, 25, 653-660.

Ogawa, S., Tank, D.W., Menon, R., Ellerman, J.M., Kim, S.-G., Merkle, H. \& Ugurbil, K. (1992) Intrinsic signal changes accompanying sensory stimulation: functional brain mapping with magnetic resonance imaging. Proc. Natl Acad. Sci. USA, 89, 5951-5955.

Oldfield, R.C. (1971) The assessment and analysis of handedness: the Edinburgh inventory. Neuropsychologia, 9, 97-113.

Penhune, V.B. \& Doyon, J. (2002) Dynamic cortical and subcortical networks in learning and delayed recall of timed motor sequences. J. Neurosci., 22, $1397-1406$.
Penhune, V.B., Zatorre, R.J. \& Evans, A.C. (1998) Cerebellar contributions to motor timing: a PET study of auditory and visual rhythm reproduction. J. Cogn. Neurosci., 10, 752-765.

Puttemans, V., Wenderoth, N. \& Swinnen, S.P. (2005) Changes in brain activation during the acquisition of a multifrequency bimanual coordination task: from the cognitive stage to advanced levels of automaticity. J. Neurosci., 25, 4270-4278.

Ramnani, N. \& Passingham, R.E. (2001) Changes in the human brain during rhythm learning. J. Cogn. Neurosci., 13, 952-966.

Rao, S., Harrington, D., Haaland, K., Bobholz, J., Cox, R. \& Binder, J. (1997) Distributed neural systems underlying the timing of movements. J. Neurosci., 17, 5528-5535.

Rao, S.M., Mayer, A.R. \& Harrington, D.L. (2001) The evolution of brain activation during temporal processing. Nature Neurosci., 4, 317-323.

Rijntjes, M., Dettmers, C., Büchel, C., Kiebel, S., Frackowiak, R.S.J. \& Weiller, C. (1999) A blueprint for movement: functional and anatomical representations in the human motor system. J. Neurosci., 19, 8043-8048.

Robertson, S.D., Zelaznik, H.N., Lantero, D.A., Bojczyk, K.G., Spencer, R.M., Doffin, J.G. \& Schneidt, T. (1999) Correlations for timing consistency among tapping and drawing tasks: evidence against a single timing process for motor control. J. Exp. Psychol: Hum. Percept. Perform., 25, 1316-1330.

Roland, P.E., Larsen, B., Lassen, N.A. \& Skinhoj, E. (1980) Supplementary area and other cortical areas in the organization of voluntary movements in man. J. Neurophysiol., 43, 118-136.

Rubia, K., Overmeyer, S., Taylor, E., Brammer, M., Williams, S.C.R., Simmons, A., Andrew, C. \& Bullmore, E.T. (2000) Functional frontalisation with age: mapping neurodevelopmental trajectories with fMRI. Neurosci. Biobehav. Rev., 24, 13-19.

Sadato, N., Campbell, G., Ibáñez, V., Deiber, M.-P. \& Hallett, M. (1996) Complexity affects regional cerebral blood flow change during sequential finger movements. J. Neurosci., 16, 2693-2700.

Sakai, K., Ramnani, N. \& Passingham, R.E. (2002) Learning of sequences of finger movements and timing: frontal lobe and action-oriented representation. J. Neurophysiol., 88, 2035-2046.

Schmahmann, J.D., Doyon, J., Toga, A.W., Petrides, M. \& Evans, A.C. (2000) MRI Atlas of the Human Cerebellum. Academic Press, San Diego.

Schubotz, R.I., Friederici, A.D. \& Von Cramon, D.Y. (2000) Time perception and motor timing: a common cortical and subcortical basis revealed by fMRI. Neuroimage, 11, 1-12.

Schubotz, R.I. \& von Cramon, D.Y. (2001) Interval and ordinal properties of sequences are associated with disctinct premotor areas. Cereb. Cortex, 11, $210-222$.

Semjen, A. \& Vos, P.G. (2002) The impact of metrical structure on performance stability in bimanual 1: 3 tapping. Psychol. Res., 66, 50-59.

Shima, K. \& Tanji, J. (2000) Neuronal activity in the supplementary and presupplementary motor areas for temporal organization of multiple movements. J. Neurophysiol., 84, 2148-2160.

Spencer, R.M., Zelaznik, H.N., Diedrichsen, J. \& Ivry, R.B. (2003) Disrupted timing of discontinuous movements by cerebellar lesions. Science, 300, $1437-1439$.

Talaraich, J. \& Tournoux, P. (1988) Co-Planar Stereotaxic Atlas of the Human Brain. Thieme, Stuttgart.

Tanji, J. (2001) Sequential organization of multiple movements: involvement of cortical motor areas. Аnпи. Rev. Neurosci., 24, 631-651.

Tanji, J. \& Shima, K. (1994) Role for supplementary motor area cells in planning several movements ahead. Nature, 371, 413-416.

Théoret, H., Haque, J. \& Pascual-Leone, A. (2001) Increased variability of paced finger tapping accuracy following repetitive magnetic stimulation of the cerebellum in humans. Neurosci. Lett., 306, 29-32.

Ullén, F. \& Bengtsson, S. (2003) Independent processing of the temporal and ordinal structure of movement sequences. J. Neurophysiol., 90, 3725-3735.

Ungerleider, L.G., Doyon, J. \& Karni, A. (2002) Imaging brain plasticity during motor skill learning. Neurobiol. Learn Mem., 78, 553-564.

Wilson, S.J., Pressing, J.L. \& Wales, R.J. (2002) Modelling rhythmic function in a musician post-stroke. Neuropsychologia, 40, 1494-1505.

Wing, A.M. (2002) Voluntary timing and brain function: an information processing approach. Brain Cogn., 48, 7-30.

Wing, A. \& Kristofferson, A. (1973) Response delays and the timing of discrete motor responses. Percept. Psychophys., 14, 5-12.

Zelaznik, H.N., Spencer, R.M.C. \& Ivry, R.B. (2002) Dissociation of explicit and implicit timing in repetitive tapping and drawing movements. J. Exp. Psychol: Hum. Percept. Perform., 28, 575-588. 